\title{
Constellation and Mapping Optimization of APSK Modulations used in DVB-S2
}

\author{
L. Jordanova \\ Department of Telecommunications, \\ Technical University of Sofia, Bulgaria \\ jordanova@tu-sofia.bg
}

\author{
L. Laskov \\ Department of Telecommunications, \\ Technical University of Sofia, Bulgaria \\ laskov@mail.com
}

\author{
D. Dobrev \\ Department of Telecommunications, \\ Technical University of Sofia, Bulgaria \\ dobrev@tu-sofia.bg
}

\begin{abstract}
This article represents the algorithms of APSK constellation and mapping optimization. The dependencies of the symbol error probability $P_{s}$ on the parameters of the 16APSK and 32APSK constellations are examined and several options that satisfy the requirements to the minimum value of $P_{s}$ are selected. Mapping optimization is carried out for the selected APSK constellations. BER characteristics of the satellite DVB-S2 channels are represented when using optimized and standard 16APSK and 32APSK constellations and a comparative analysis of the results achieved is made.
\end{abstract}

Keywords-satellite DVB channel; M-ary APSK constellation and mapping; concateneted BCH-LDPC codes; DVB-S2 channel noise immunity; $Q E F$ reception

\section{INTRODUCTION}

In the contemporary Digital Video Broadcasting (DVB) systems it is necessary to provide Quasi-Error-Free (QEF) reception while the values of the Carrier to Noise Ratio (CNR) parameter are relatively low and the encoding and decoding equipment is not very complex [1]. The term "Quasi-ErrorFree" means that the bit error rate (BER) at the input of the MPEG demultiplexer is less than $10^{-10}$ to $10^{-11}$.

The requirement of contemporary communication systems to provide a higher rate of transmitted data requires the application of modulation methods with a greater spectral efficiency. When an $\mathrm{M}$-ary modulation technique of higher order is used then both the spectral efficiency and the bit rate are increased but, in turn, the channel noise immunity is decreased.

In satellite systems, apart from noise and interference, the channel nonlinearity also causes a problem. The nonlinearity exists because the working mode of the power amplifier board is set near the saturation point in order to reach the maximum level for the transmitted signal. The nonlinear signal distortions, similarly to the noise and interference in the radio channel, are the reasons for the increase of error probability.

An essential requirement to the satellite DVB systems is ensuring high power efficiency without excessively penalizing the spectrum efficiency. This is achieved by using noise resistant types of modulation and effective channel codes. As known, the APSK modulation provides great resistance to nonlinear distortion in a radio channel $[2,3]$, which is the reason why this modulation is used in the second-generation satellite DVB systems. The 16APSK and 32APSK have been proposed in the DVB-S2 standard [4] and their performances have been widely investigated over the AWGN channel, by considering typical satellite scenarios, also in the presence of High Power Amplifiers (HPAs). In order to enhance the noise immunity of a DVB-S2 channel, a concatenated error protection of Bose-Chaudhuri-Hocquenghem $(\mathrm{BCH})$ outer code and Low Density Parity Check (LDPC) inner code was chosen $[4,5]$.

The aim of this paper is to study the influence of the 16APSK and 32APSK constellation parameters and mapping on the noise immunity of satellite DVB channels.

\section{ALGORITHM FOR APSK CONSTELLATION AND MAPPING OPTIMIZATION}

The APSK constellation consists of a $N$ number of concentric circles, where the $k$-th circle contains $n_{k}$ signal points. Each of the circles in the constellation is characterized by a primary phase shift $\varphi_{k}$ and radius $r_{k}$. In the general case, the APSK constellation can be described as follows:

$$
\psi=\left\{\begin{array}{cc}
r_{1} \cdot \exp \left[j\left(\varphi_{1}+\frac{2 \pi}{n_{1}} n\right)\right] & n=0,1, \ldots, n_{1}-1 \\
r_{2} \cdot \exp \left[j\left(\varphi_{2}+\frac{2 \pi}{n_{2}} n\right)\right] & n=0,1, \ldots, n_{2}-1 \\
\ldots & \ldots \\
r_{N} \cdot \exp \left[j\left(\varphi_{N}+\frac{2 \pi}{n_{N}} n\right)\right] & n=0,1, \ldots, n_{N}-1
\end{array}\right\}
$$

For convenience, instead of the radiuses, their ratios relative to the radius of the innermost circle $-\gamma_{k}=r_{(k+1)} / r_{1}$ may be used. The APSK modulation is usually denoted as $n_{1}-n_{2}-\ldots$ APSK.

Figure 1 and Figure 2 show 16APSK and 32APSK standard constellations that are used in the DVB-S2 systems. The parameters of these constellations are as follows: $N=2, n_{1}=4$, 
$n_{2}=12, \varphi_{1}=45^{\circ}, \varphi_{2}=15^{\circ}$ and $\gamma_{1}=r_{2} / r_{1}=2.6$ (for 16APSK) and $N=3, n_{1}=4, n_{2}=12, n_{3}=16, \varphi_{1}=45^{\circ}, \varphi_{2}=15^{\circ}, \varphi_{3}=0^{\circ}, \gamma_{1}=r_{2} / r_{1}=2.54$ and $\gamma_{2}=r_{3} / r_{1}=4.33$ (for 32APSK). These constellations are determined to be optimal at an LDPC code rate of 8/9 and BCH code rate of 0.983 .

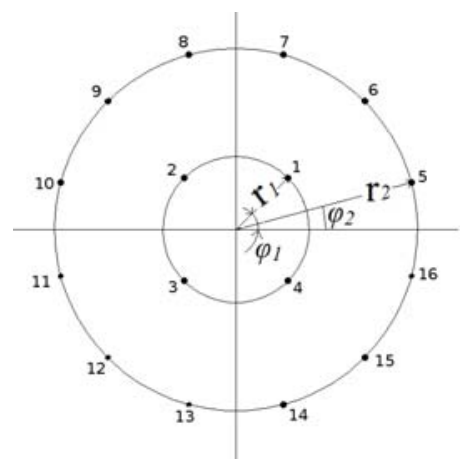

Fig. 1. Standard 4+12 APSK constellation

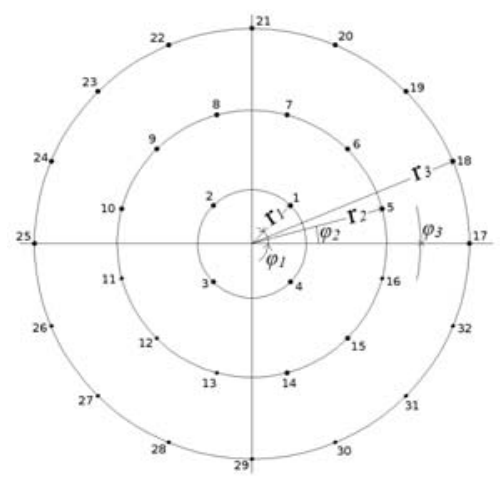

Fig. 2. 32APSK Constellation

There are several known algorithms for the optimization of APSK constellations [6, 7]. The aim of the optimization, presented in this paper, is to provide minimum symbol error probability. After processing the basic expressions, given in [8], the following dependence for the determination of the symbol error probability $P_{s}$ was obtained:

$$
P_{s} \leq \frac{1}{M} \sum_{i=1}^{M} \sum_{j=1, j \neq i}^{M} P\left(s_{i} \rightarrow s_{j}\right)=\frac{1}{M} \sum_{i=1}^{M} \sum_{j=1, j \neq i}^{M} \frac{1}{2} \operatorname{erfc}\left(\frac{d_{i j}}{2 \sqrt{N_{0}}}\right)
$$

In this expression, $P\left(s_{i} \rightarrow s_{j}\right)$ denotes the probability that instead of the $i$-th symbol the $j$-th one is accepted, $M$ is the modulation order, $N_{0}$ is the noise power density and $d_{i j}$ is the Euclidean distance between the $i$-th and $j$-th points of the constellation. The value of the error complementary function is obtained by [9]:

$$
\operatorname{erfc}(x) \approx \frac{1}{x \sqrt{\pi}} \cdot \exp \left(-x^{2}\right)
$$

In order to determine the Euclidean distance between two points which define the $i$-th and $j$-th positions of the APSK signal vector, we can use the cosines theorem, i.e.

$$
d_{i j}=\sqrt{r_{p(i)}^{2}+r_{q(j)}^{2}-2 \cdot r_{p(i)} \cdot r_{q(j)} \cdot \cos \Theta_{i j}}
$$

where $r_{p(i)}$ and $r_{q(j)}$ are the radiuses of the circles where the two points are located and $\theta_{i j}$ is the angle between the signal vectors studied. The value of $\theta_{i j}$ is derived by [7]:

$$
\Theta_{i j}=\left|\left(\varphi_{p}-\varphi_{q}\right)+2 \pi\left(\frac{i-1}{n_{p}}-\frac{j-1}{n_{q}}\right)\right|(5),
$$

where $\varphi_{p}$ and $\varphi_{q}$ denote the relative phase shifting of the signal points located on the $p$-th and $q$-th circles, $n_{p}$ and $n_{q}$ is the number of these points.

The relation between the radiuses of the circles in the APSK constellation and the energy per symbol $E_{s}$ can be described by the following dependence:

$$
E_{s}=\frac{n_{1} r_{1}^{2}+n_{2} r_{2}^{2}+\ldots+n_{N} r_{N}^{2}}{M}=\frac{r_{1}^{2}\left(n_{1}+n_{2} \gamma_{1}^{2}+\ldots+n_{N} \gamma_{N-1}^{2}\right)}{M}
$$

where $\gamma_{i}=r_{(i+1)} / r_{1}$.

The mathematical model described allows the optimization of the following parameters of APSK constellations: the number of phase states $n_{k}$ of the signal vector with amplitude $r_{k}$, the ratios between the amplitudes of the signal vector $\gamma_{(k-1)}=r_{k} / r_{1}$ and the relative phase shifting of the symbol points $\varphi_{k}$, where $k=1,2, \ldots N$. For the optimization of the APSK constellation parameters, we need to find the minimum value of the functional dependence which is presented by (1).

In order to evaluate the efficiency of the power used, the PAPR parameter (Peak to Average Power Ratio) is used. It is the ratio between the maximum and the average signal power $s(t)$ for a period $T$ and is obtained using the expression [10]:

$$
P A P R=\frac{\max _{t \in[0, T]}|s(t)|^{2}}{\frac{1}{T} \int_{0}^{T}|s(t)|^{2} d t}=\frac{M r_{N}^{2}}{\sum_{i=1}^{M} r_{p(i)}^{2}}
$$

where $r_{N}$ denotes the radius of the outermost circle, and $r_{p(i)}$ denotes the radius of the circle on which the $i$-th signal point is located.

In order to ensure the minimum bit error probability $P_{b}$, it is required to select an appropriate combination of $\log _{2} \mathrm{M}$ bits (mapping) for each signal point of the APSK constellation. The following dependence can be used when mapping optimization is carried out:

$$
\begin{aligned}
& P_{b} \leq \frac{1}{M} \sum_{i=1}^{M} \sum_{j=1, j \neq i}^{M} \frac{h_{i j}}{\log _{2} M} P\left(s_{i} \rightarrow s_{j}\right)=\ldots \\
& \ldots=\frac{1}{M} \sum_{i=1}^{M} \sum_{j=1, j \neq i}^{M} \frac{h_{i j}}{2 \log _{2} M} \operatorname{erfc}\left(\frac{d_{i j}}{2 \sqrt{N_{0}}}\right)
\end{aligned}
$$

where $h_{i j}$ is the Hamming distance between the $i$-th and $j$-th signal points. 


\section{PARAMETERS OF THE OPTIMIZED 16APSK CONSTELLATIONS}

In order to determine the optimal parameters of the 16APSK constellation, different combinations of parameters $n_{1}$, $n_{2}$ and $\varphi_{1}$ were used and for each of them, the minimum of the functional dependence of the symbol error probability $P_{s}$ on parameters $\varphi_{2}$ and $\gamma_{1}$ was established. The analysis of the results achieved shows that the initial phase shift $\varphi_{2}$ of the signal points of the outer circle does not impact considerably the $P_{s}$. Therefore, in this study it is assumed that $\varphi_{2}=0^{\circ}$.

Figure 3 shows the dependences of the symbol error probability on the ratio between the outer and inner circles of the 16APSK constellation $\gamma_{1}$ for four combinations of parameters $n_{1}, n_{2}$ and $\varphi_{1}$, for which the best results have been achieved. These dependences have been obtained at an energy per symbol to noise power density ratio of $E_{s} / N_{0}=12 \mathrm{~dB}$. When determining the value of $E_{s} / N_{0}$ parameter, the real Carrier to Noise Ratio (CNR) at the input of the satellite receiver has been considered (usually varies from 9 to $12 \mathrm{~dB}$ ).

The values of the parameters of the four studied constellations at which a minimum symbol error probability is achieved are given in Table I. The table also shows the calculated values of the PAPR parameter for the evaluation of the power efficiency of these constellations. Obviously, the highest radio channel noise immunity can be ensured at using the fourth 16APSK constellation (CN4), but in that case the power efficiency of the system is the lowest. The best power efficiency is achieved at the first of the compared constellations (CN1), but it would provide the lowest radio channel noise immunity. So when choosing an APSK constellation, a compromise between the noise immunity and the power efficiency is to be made.

The mapping optimization carried out demonstrates that there is more than one possible combination of $m=\log _{2}(M)$ bits corresponding to the signal points, which provide a minimum of the functional dependence. The optimization is based on two constraints that define the permissible number of differing bits for two adjacent signal points $\zeta$. The first one relates to the signal points of one and the same circle, and for them $\zeta=1$. Only one breach of this rule is allowed, and then $\zeta=2$. The second constraint requires that the value of the parameter $\zeta$ for the adjacent signal points from different circles is not higher than 2 .

For each of the 16APSK constellations presented in Table I, one of the possible $m=4$ bits combinations at which a minimum value of bit error probability $P_{b}$ is achieved is shown in Table II.

TABLE I. PARAMETERS OF THE OPTIMAL 16APSK CONSTELLATION.

\begin{tabular}{|c|c|c|c|c|c|}
\hline $\begin{array}{c}\text { Constellation } \\
\text { Number }(\mathbf{C N})\end{array}$ & $\boldsymbol{\gamma}_{\mathbf{1}}$ & $\mathbf{n}_{\mathbf{1}}, \mathbf{n}_{\mathbf{2}}$ & $\boldsymbol{\varphi}_{\mathbf{1}}$ & $\mathbf{P}_{\mathbf{s} \text { min }}$ & PAPR \\
\hline CN1 & 2.99 & 3,13 & 60 & 0.1524 & 1.197 \\
\hline CN2 & 2.61 & 4,12 & 45 & 0.1291 & 1.266 \\
\hline CN3 & 2.37 & 5,11 & 36 & 0.1175 & 1.335 \\
\hline CN4 & 2.20 & 6,10 & 30 & 0.1166 & 1.403 \\
\hline
\end{tabular}

TABLE II. OPTIMAL SYMBOL MAPPING

\begin{tabular}{|c|c|c|c|c|}
\hline $\begin{array}{c}\text { Symbol } \\
\text { Point No }\end{array}$ & CN1 & CN2 & CN3 & CN4 \\
\hline 1 & 0000 & 1100 & 0000 & 0000 \\
\hline 2 & 1000 & 1110 & 1000 & 1000 \\
\hline 3 & 1100 & 1111 & 1100 & 1100 \\
\hline 4 & 0100 & 1101 & 1110 & 1110 \\
\hline 5 & 0110 & 0100 & 1010 & 0110 \\
\hline 6 & 0010 & 0000 & 0010 & 0100 \\
\hline 7 & 0011 & 1000 & 0011 & 0011 \\
\hline 8 & 0001 & 1010 & 0001 & 0010 \\
\hline 9 & 1001 & 0010 & 1001 & 1010 \\
\hline 10 & 1011 & 0110 & 1101 & 1011 \\
\hline 11 & 1010 & 0111 & 0101 & 1001 \\
\hline 12 & 1110 & 0011 & 0100 & 1101 \\
\hline 13 & 1111 & 1011 & 0110 & 1111 \\
\hline 14 & 1101 & 1001 & 0111 & 0111 \\
\hline 15 & 0101 & 0001 & 1111 & 0101 \\
\hline 16 & 0111 & 0101 & 1011 & 0001 \\
\hline
\end{tabular}

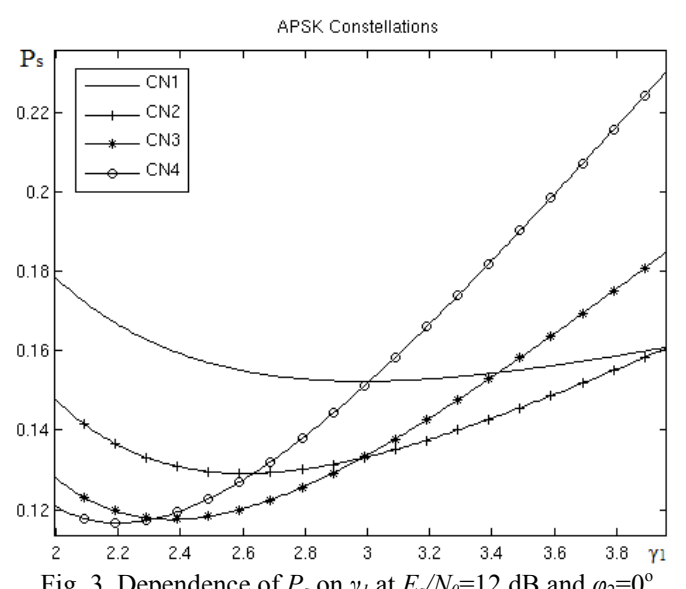

Fig. 3. Dependence of $P_{s}$ on $\gamma_{1}$ at $E_{s} / N_{0}=12 \mathrm{~dB}$ and $\varphi_{2}=0^{\circ}$

\section{PARAMETERS OF THE OPTIMIZED 32APSK CONSTELLATIONS}

At determining the optimum parameters of the 32APSK constellation, different combinations of parameters $n_{1}, n_{2}, n_{3}$, $\varphi_{1}, \varphi_{2}$ and $\varphi_{3}$ have been used, and for each of them the values of $\gamma_{1}$ and $\gamma_{2}$ for which the symbol error probability $P_{s}$ was minimal were determined. Figure 4 shows the dependence of $P_{s}$ on $\gamma_{1}$ and $\gamma_{2}$ of the $4+12+16$ APSK constellation, for which the most favorable results are achieved. The parameters of this constellation, denoted by $\mathrm{CN} 1$, as well as the calculated values of $P_{s}$ and PAPR are given in Table III.

Similar studies have been carried out for other combinations of the 32APSK constellation parameters and after an analysis of the results achieved another four options have been selected and marked as $\mathrm{CN} 2, \mathrm{CN} 3, \mathrm{CN} 4$ and $\mathrm{CN} 5$. Figure 5 and 6 show the dependences of the symbol error probability $P_{s}$ on parameters $\gamma_{1}$ and $\gamma_{2}$ of the $4+8+20$ APSK (CN2) and 5+11+16 APSK (CN3) constellations. It is assumed that $E_{S} / N_{0}=15 \mathrm{~dB}$ for these dependences. As seen from Table III, the $5+11+16$ APSK constellation would provide the minimum symbol error probability, and the $4+8+20$ APSK the most efficient use of the transmitter's power. 
The mapping optimization carried out shows that a minimum of the functional dependence can be achieved for various bit combinations in the 32 symbol points of the studied APSK constellations. The combinations, for which the lowest values of the $P_{b}$ parameter are obtained, are given in Table IV.

\section{NOISE IMMUNITY OF THE DVB-S2 CHANNEL}

In order to assess the noise immunity of the DVB-S2 channel, the dependence of bit error probability $P_{b}$, respectively BER at the output of the channel decoder, on the energy per symbol to noise power density ratio $E_{b} / N_{0}$ has been used. The expressions for determining the $P_{b}$ are given in [11], and the values of $E_{b} / N_{0}$ parameter are calculated by:

$$
\frac{E_{s}}{N_{0}}=\frac{E_{b}}{N_{0}}+10 \cdot \lg (m)+10 \cdot \lg \left(R_{\mathrm{LDPC}}\right)+10 \cdot \lg \left(R_{\mathrm{BCH}}\right)
$$

where $m=\log _{2}(M)$ denotes the number of bites in one symbol, and $R_{\mathrm{LDPC}}$ and $R_{\mathrm{BCH}}$ are the rates of the channel codes used.

Figure 7 shows the dependences of BER on the $E_{b} / N_{0}$ of DVB-S2 channels at using the four studied constellations and the standard 16APSK one. These dependences have been obtained at channel code rates $R_{\mathrm{LDPC}}=8 / 9$ and $R_{\mathrm{BCH}}=0.983$. The values of the parameter $E_{b} / N_{0}$, for which the bit error rate is $10^{-11}$, are given in Table $\mathrm{V}$. As it is evident in Table $\mathrm{V}$, the best radio channel noise immunity can be provided when the fourth APSK constellation is used. In comparison with the APSK constellation which is standard for DVB-S2, the achieved benefit is $0.1 \mathrm{~dB}$.

The assessment of the noise immunity of DVB-S2 channels at using the studied constellations and the standard 32APSK one can be derived from Figure 8. In this case also, it is assumed that the channel code rates are $\mathrm{RLDPC}=8 / 9$ and $\mathrm{RBCH}=0.983$. The values of the parameter $\mathrm{Eb} / \mathrm{N} 0$, for which the bit error rate is $10^{-11}$, are given in Table VI.

Obviously, the best radio channel noise immunity can be provided when the first 32APSK constellation is used. In comparison with the 32APSK constellation which is standard for DVB-S2, the achieved benefit is $0.085 \mathrm{~dB}$.

\section{CONCLUSION}

The analytic and graphic dependences presented in this paper make it possible for the parameters and mapping of 16APSK and 32APSK constellations to be determined. These dependences can be used in any other communication system in which such types of modulations are applied. The researches on the noise immunity of DVB-S2 channels, for the formation of which the studied 16APSK and 32APSK constellations have been used, show that there are several options for achieving similar results to those set out in the standard.

\section{ACKNOWLEDGMENT}

The research described in this paper is supported by the Bulgarian National Science Fund under the contract No DDVU 02/74/2010.

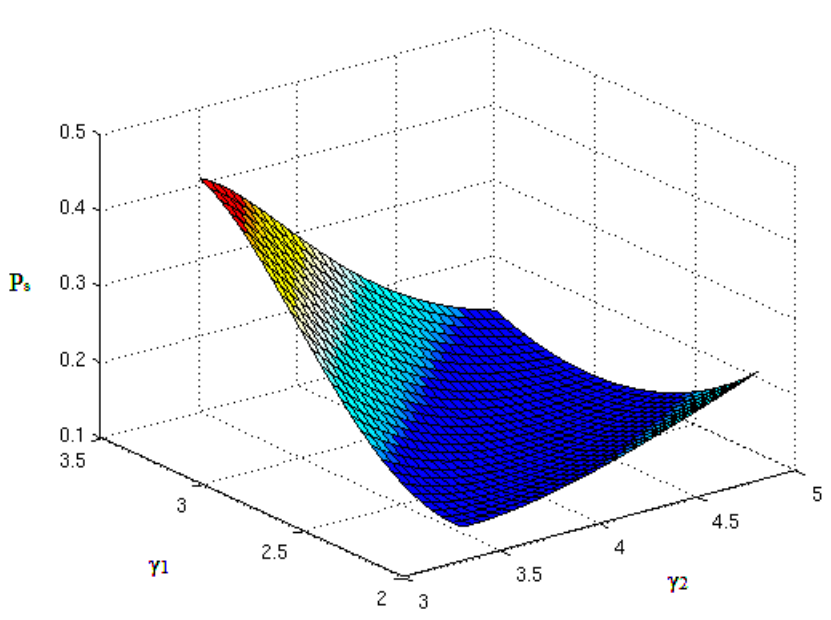

Fig. 4. Dependence of $P_{s}$ on $\gamma_{1}$ and $\gamma_{2}$ of $4+12+16$ APSK constellation at $E_{s} / N_{0}=15 \mathrm{~dB}$

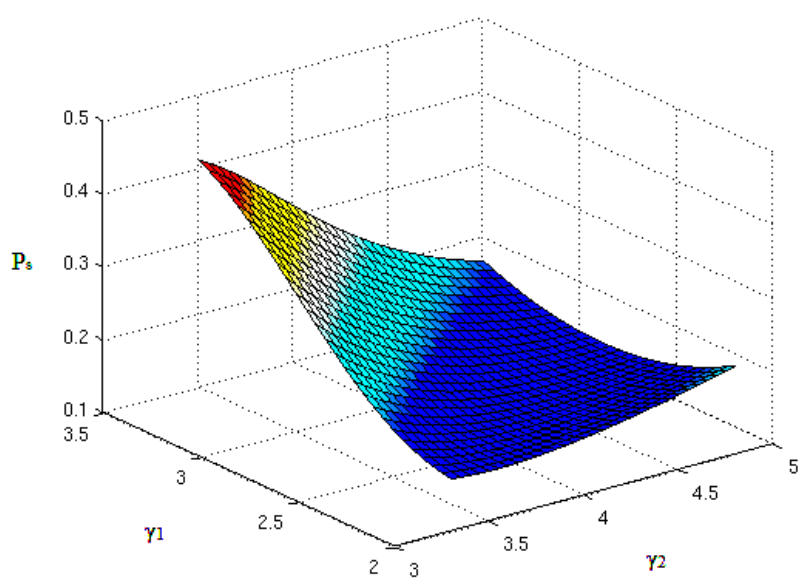

Fig. 5. Dependence of $P_{s}$ on $\gamma_{1}$ and $\gamma_{2}$ of $4+8+20$ APSK constellation

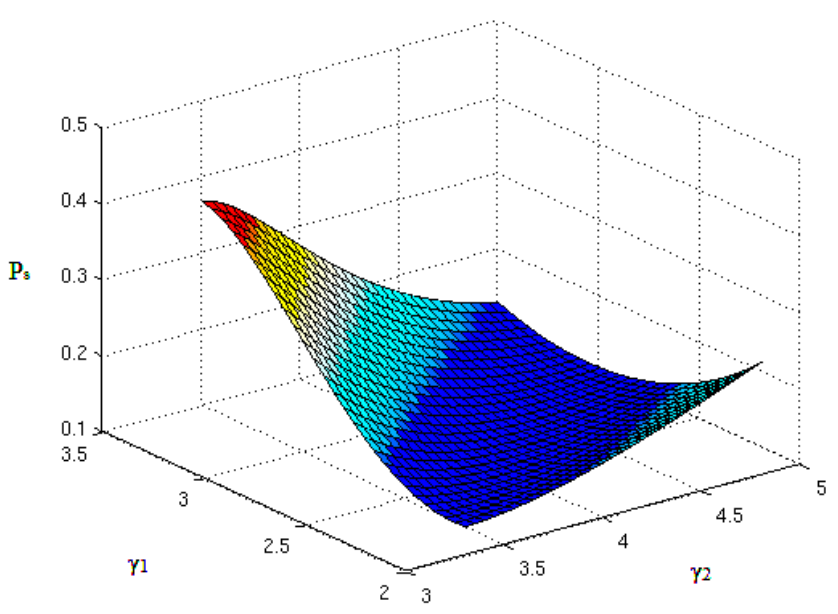

Fig. 6. Dependence of $P_{s}$ on $\gamma_{1}$ and $\gamma_{2}$ of $5+11+16$ APSK constellation 
TABLE III. PARAMETERS OF THE OPTIMAL 32APSK CONSTELLATION.

\begin{tabular}{|c|c|c|c|c|c|}
\hline $\begin{array}{c}\text { Constellation } \\
\text { Number }(\mathbf{C N})\end{array}$ & $\mathbf{C N 1}$ & $\mathbf{C N 2}$ & $\mathbf{C N 3}$ & $\mathbf{C N 4}$ & $\mathbf{C N 5}$ \\
\hline $\boldsymbol{n}_{\boldsymbol{1}}, \boldsymbol{n}_{\mathbf{2}}, \boldsymbol{n}_{\mathbf{3}}$ & $4,12,16$ & $4,8,20$ & $5,11,16$ & $5,10,17$ & $6,10,16$ \\
\hline$\gamma_{\boldsymbol{1}}$ & 2.54 & 2.32 & 2.3 & 2.26 & 2.13 \\
\hline$\gamma_{\boldsymbol{2}}$ & 4.23 & 4.07 & 3.75 & 3.73 & 3.41 \\
\hline $\boldsymbol{\varphi}_{\boldsymbol{1}}$ & 45 & 45 & 36 & 36 & 30 \\
\hline $\boldsymbol{\varphi}_{\boldsymbol{2}}$ & 0 & 22.5 & 16.36 & 18 & 0 \\
\hline $\boldsymbol{\varphi}_{\boldsymbol{3}}$ & 11.25 & 0 & 11.25 & 0 & 0 \\
\hline $\boldsymbol{P}_{\boldsymbol{s}}$ & 0.1365 & 0.1644 & 0.1331 & 0.1368 & 0.1348 \\
\hline PAPR & 1.557 & 1.401 & 1.561 & 1.522 & 1.567 \\
\hline
\end{tabular}

TABLE IV. OPTIMAL SYMBOL MAPPING

\begin{tabular}{|c|c|c|c|c|c|}
\hline $\begin{array}{l}\text { Symbol } \\
\text { Point № }\end{array}$ & CN1 & CN2 & CN3 & CN4 & CN5 \\
\hline 1 & 00000 & 00000 & 00000 & 00000 & 00000 \\
\hline 2 & 10000 & 10000 & 10000 & 10000 & 10000 \\
\hline 3 & 11000 & 11000 & 11000 & 11000 & 11000 \\
\hline 4 & 01000 & 01000 & 11100 & 11100 & 11100 \\
\hline 5 & 00001 & 00001 & 10100 & 01100 & 01100 \\
\hline 6 & 00101 & 00101 & 00100 & 01001 & 01000 \\
\hline 7 & 00100 & 00100 & 00110 & 00001 & 01001 \\
\hline 8 & 10100 & 10100 & 00010 & 10001 & 00001 \\
\hline 9 & 10101 & 11100 & 10010 & 11001 & 10001 \\
\hline 10 & 10001 & 11101 & 11010 & 11011 & 11001 \\
\hline 11 & 11001 & 11001 & 01010 & 11010 & 11011 \\
\hline 12 & 11101 & 01001 & 01000 & 11110 & 11010 \\
\hline 13 & 11100 & 00011 & 01100 & 01110 & 11110 \\
\hline 14 & 01100 & 10011 & 01110 & 01010 & 01110 \\
\hline 15 & 01101 & 10001 & 11110 & 01000 & 01010 \\
\hline 16 & 01001 & 10101 & 10110 & 01101 & 01011 \\
\hline 17 & 00011 & 10111 & 00101 & 01111 & 01111 \\
\hline 18 & 00111 & 00111 & 00111 & 00111 & 01101 \\
\hline 19 & 00110 & 00110 & 00011 & 00101 & 00101 \\
\hline 20 & 10110 & 00010 & 00001 & 10101 & 10101 \\
\hline 21 & 10111 & 10010 & 10001 & 11101 & 11101 \\
\hline 22 & 10011 & 10110 & 10011 & 11111 & 11111 \\
\hline 23 & 10010 & 11110 & 11011 & 10111 & 10111 \\
\hline 24 & 11010 & 01110 & 01011 & 10011 & 10011 \\
\hline 25 & 11011 & 01100 & 01001 & 10010 & 10010 \\
\hline 26 & 11111 & 01101 & 11001 & 10110 & 10110 \\
\hline 27 & 11110 & 01111 & 11101 & 10100 & 10100 \\
\hline 28 & 01110 & 11111 & 01101 & 00100 & 00100 \\
\hline 29 & 01111 & 11011 & 01111 & 00110 & 00110 \\
\hline 30 & 01011 & 11010 & 11111 & 00010 & 00010 \\
\hline 31 & 01010 & 01010 & 10111 & 00011 & 00011 \\
\hline 32 & 00010 & 01011 & 10101 & 01011 & 00111 \\
\hline
\end{tabular}

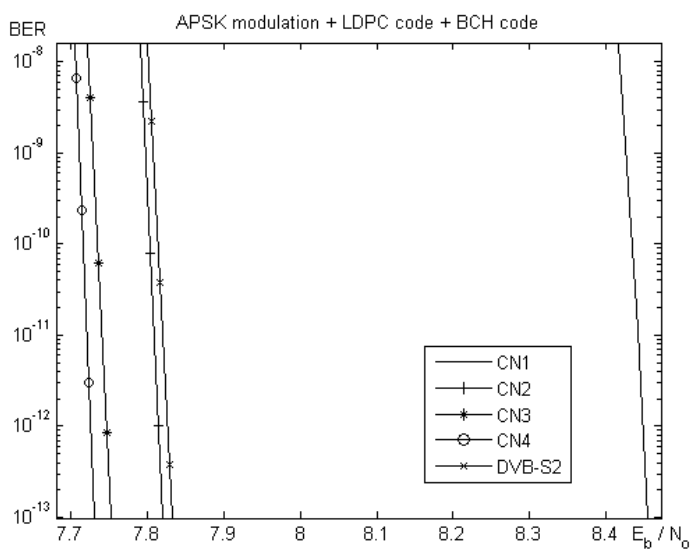

Fig. 7. BER characteristics of 16 APSK channels
TABLE V. VALUES OF $E_{B} / N_{0}$ THAT PROVIDED BER $=10^{-11}$ (16APSK)

\begin{tabular}{|c|c|c|c|c|}
\hline CN1 & CN2 & CN3 & CN4 & DVB-S2 \\
\hline $8.443 \mathrm{~dB}$ & $7.809 \mathrm{~dB}$ & $7.741 \mathrm{~dB}$ & $7.721 \mathrm{~dB}$ & $7.821 \mathrm{~dB}$ \\
\hline
\end{tabular}

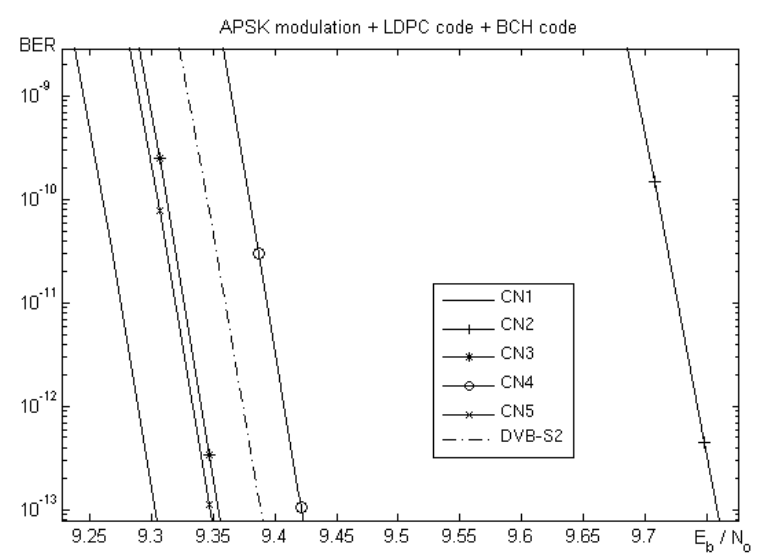

Fig. 8. BER characteristics of 32APSK channels

TABLE VI. VALUES OF $E_{B} / N_{0}$ THAT PROVIDED BER $=10^{-11}$ (32APSK)

\begin{tabular}{|c|c|c|c|c|c|}
\hline CN1 & CN2 & CN3 & CN4 & CN5 & DVB-S2 \\
\hline $9.275 \mathrm{~dB}$ & $9.727 \mathrm{~dB}$ & $9.326 \mathrm{~dB}$ & $9.393 \mathrm{~dB}$ & $9.319 \mathrm{~dB}$ & $9.36 \mathrm{~dB}$ \\
\hline
\end{tabular}

\section{REFERENCES}

[1] ETSI EN 300421 V1.1.2, Digital video broadcasting (DVB): Framing structure, channel coding and modulation for $11 / 12 \mathrm{GHz}$ satellite services, European Telecommunications Standards Institute, 1997

[2] A. Morello, V. Mignone, "DVB-S2: The second generation standard for satellite broad-band services", Proceedings of the IEEE, Vol. 94, No. 1, pp. 210-226, 2006

[3] R. Muller, U. Wachsmann, J. Huber, "Multilevel coding for peak power limited complex Gaussian channels”, IEEE International Symposium on Information Theory, Ulm, Germany, 1997

[4] ETSI EN 302307 V121, Digital video broadcasting (DVB): Second generation framing structure, channel coding, and modulation systems for broadcasting, interactive services, news gathering and other broadband satellite applications, European Telecommunications Standards Institute, 2009

[5] ETSI TR 102 376, User guidelines for the second generation system for broadcasting, interactive services, news gathering and other broadband satellite applications (DVB-S2), European Telecommunications Standards Institute, 2005

[6] R. de Gaudenzi, A. Guillen i Fabregas, A. Martinez, "Turbo-coded APSK modulations design for satellite broadband communications", International Journal of Satellite Communications and Networking, , Vol. 24, pp. 261-281, 2006

[7] K. Liolis, R. Gaudenzi, N. Alagha, A. Martinez, A. Fabregas, "Amplitude phase shift keying constellation design and its applications to satellite digital video broadcasting", in: Digital Video, ISBN 978-9537619-70-1, InTech, 2010

[8] O. Afelumo, A. Awoseyila, B. Evans, "Simplified evaluation of APSK error performance", Electronics Letters, Vol. 48, No. 14, pp. 886-888, 2012

[9] B. Sklar, Digital communication: Fundamentals and applications, Prentice Hall, 2001

[10] M. Baldi, F. Chiaraluce, A. de Angelis, R. Marchesani, S. Schillaci, "A comparison between APSK and QAM in wireless tactical scenarios for land mobile systems", EURASIP Journal on Wireless Communications and Networking, article number: 317, pp. 1-14, 2012 
[11] L. Jordanova, L. Laskov, D. Dobrev, "Influence of BCH and LDPC code parameters on the BER characteristic of satellite DVB channels", Engineering, Technology \& Applied Science Research, Vol. 4, No. 1, pp. 591-595, 2014

[12] R. de Gaudenzi, A. Guillen i Fabrigas, A. Martinez, "Performance analysis of turbo-coded APSK modulations over nonlinear satellite channels", IEEE Transactions on Wireless Communications, Vol. 5, No. 9, pp. 2396-2407, 2006

[13] S. Jonson, Iterative error correction: Turbo, low-density parity-check and repeat-accumulate codes, Cambridge University Press, 2010

[14] M. Luby, M. Mitzenmachery, M. Shokrollahiz, D. Spielmanx, "Analysis of low density codes and improved designs using irregular graphs", Proceedings of the 30th ACM Symposium on Theory of Computing, pp. 249-258, New York, 1998

[15] W. Fisher, Digital video and audio broadcasting technology, Springer, 2008

[16] C. Thomas, M. Weidner, S. Durrani, "Digital amplitude-phase keying with M-ary alphabets", IEEE Transactions on Communications, Vol. 22, No. 2, pp. 168-180, 1974

[17] C. Berrou, Codes and turbo codes. Springer, 2010

[18] M. Eroz, F. Sun, L. Lee, "DVB-S2 low density parity check codes with near Shannon limit performance", International Journal of Satellite Communications and Networking, Vol. 22, No. 3, pp. 269-279, 2004

[19] L. Ippolito, Satellite communications system engineering, John Wiley \& Sons Ltd., 2008

[20] Newtec EL470, IP satellite modem, www.newtec.eu.

[21] K. Liolis, N. Alagha, "On 64-APSK constellation design optimization", Proceedings of 10th International Workshop on Signal Processing for Space Communications, Rhodes, Greece, 2008.

[22] W. Sung, S. Kang, P. Kim, D. Chang, "Performance analysis of APSK modulation for DVB-S2 transmission over nonlinear channels", International Journal of Satellite Communications and Networking, Vol. 27 ,pp. 295-311, 2009 